\title{
Gestaltungsdomänen des Kostenmanagements
}

\author{
Von Michael Reiß und Hans Corsten
}

\section{A Gestaltungskontext des Kostenmanagements}

Kostenmanagement bezeichnet eine Gestaltung der Programme, Potentiale und Prozesse in einer Unternehmung nach Kostenkriterien. Mit anderen Worten werden im Rahmen eines Kostenmanagements so unterschiedliche Entscheidungen wie die über den Diversifikationsgrad (Breite) und die Differenziertheit (Sorten- bzw. Variantenvielfalt) des Produktprogramms, über die Fertigungstiefe (Eigenerstellung oder Fremdbezug), über alternative Produktionsverfahren und logistische Prozesse sowie über die Anpassung von Kapazitäten und Betriebsgrößen, die Einsatzmengen von Materialien bzw. über eine Werkstoffsubstitution durchweg mit Blick auf die jeweils relevanten Kosten gefällt. Offensichtlich handelt es sich beim Kostenmanagement um eine klassische Domäne der Unternehmungsführung.

Mehrere breit angelegte aktuelle Trends sorgen dafür, da $B$ diese Sparte des Managements nicht an Bedeutung einbüßt, sondern eher an Stellenwert gewinnt. So läßt sich etwa die in allen Branchen feststellbare Ertragsschwäche von wachsenden Unternehmungen auf ein defizitäres Kostenmanagement zurückführen. In dem Bemühen um schnelles Wachstum der Umsätze bzw. relativen Marktanteile wird oft die Kostenseite vernachlässigt. Umgekehrt ist im Falle einer rückläufigen Auftragslage ein Kostenmanagement erforderlich, um die remanenten Kosten, gewissermaßen die flexibilitätshemmende Kehrseite der Expansion, zu handhaben. Aber nicht nur die Höhe der Kosten und das oft wenig anpassungsfähige Kostenverhalten machen ein systematisches Kostenmanagement zum Muß-Bestandteil eines Management-Systems. Im Zuge des Wachstums auf der Basis kapitalintensiver Technologien werden auch die Kostenstrukturen immer schwerer beherrschbar. Hohe Fixkostenanteile entziehen immer mehr Kostenbestandteile einer produktorientierten Beeinflussung. Der Gestaltungsspielraum des konventionellen Kostenmanagers schrumpft häufig auf ein Nischen-Format zusammen. Die Komplexität der Produkte und Prozes- 
se sowie die verstärkte Integration der Unternehmungsprozesse ziehen eine Ausdehnung des indirekten Bereichs nach sich, und zwar nicht nur in der Zentralverwaltung, sondern auch in den fertigungsnahen Bereichen. Diese Veränderungen in den Kostenstrukturen haben bekanntlich eine Anpassung der Kostenrechnungssysteme erzwungen, weil die traditionellen Verfahren auf der Basis des Zurechnungs- und Zuschlagsprinzips immer größere Verfälschungen der Kosteninformationen provozieren. Sie müssen darüber hinaus auch mit neuen Konzepten eines Kosten-Strukturmanagements beantwortet werden.

Um den genannten branchenübergreifenden Entwicklungen gerecht zu werden, muB das Kostenmanagement eine strategische Ausrichtung erhalten. In diesem Sinne sollte Kostenmanagement nicht primär als Risiko-Management (mitunter sogar ,Krisenmanagement"), sondern als Chancen-Management betrieben werden. Zu diesem Zweck muß allerdings ein Wechsel vom reaktiven zum prospektiven Kostenmanagement erfolgen. Prospektives Kostenmanagement antizipiert zukünftige Handlungsbedarfe und schafft vorsorglich die notwendigen Handlungsspielräume für Kostenanpassungen, beispielsweise in Form von flexiblen Mitarbeitern, Fertigungssystemen oder Vereinbarungen mit Zulieferern. Die Kosten bilden dabei definitionsgemä $\beta$ die intendierten Gestaltungs- bzw. Beeinflussungsobjekte (,targets“) eines Kostenmanagements. Kosten sind ihrerseits Wirkungen bestimmter Kosteneinflußgrößen. Die unmittelbaren Angriffspunkte für einen Eingriff müssen folglich im Bereich dieser Kostenbestimmungsfaktoren lokalisiert werden. Einen ersten Überblick über die Vielfalt dieser Kostenverursachungsfaktoren geben die traditionellen Hauptkosteneinflußgrößen, d. h. die Betriebsgröße, das Produktionsprogramm, der Produktionsablauf (allgemein: Prozesse), die Qualitäten, Mengen und Preise der Inputgüter (Kostenarten) sowie die absolute oder relative Beschäftigung (Outputmenge).

Die Gesamtheit aller Aktivitäten eines Kostenmanagements läßt sich nach zwei Kriterien in überschaubare Aktivitätsfelder (Gestaltungsdomänen) unterteilen:

- Zum einen nach dem Gestaltungsobjekt in die Domänen des Niveau-, Verlaufsbzw. Strukturmanagements (vgl. B). Kostenniveau bezeichnet die Höhe der Kosten, Kostenverlauf die mehr oder weniger stark ausgeprägte Reagibilität der Kosten gegenüber bestimmten Kosteneinflußgrößen und Kostenstruktur schließlich die Zusammensetzung der Kosten aus unterschiedlichen Kosten..blöcken“, -kategorien bzw. -arten.

- Zum anderen nach den Gestaltungsfunktionen in die Managementdomänen der Diagnose, der Planung und der Implementierung (vgl. C).

Wie bei jeder analytischen Aufteilung muß auch hier der Vorteil der besseren Überschaubarkeit der Materie erkauft werden durch den Nachteil einer künstlichen Trennung von zusammengehörigen Aktivitäten. 


\section{B Gestaltungsobjekte: Niveau, Verlauf und Struktur der Kosten}

\section{Kostenniveau-Management}

Ziel dieser Strategie ist eine Reduzierung des allgemeinen Kostenniveaus. Hier kommen meist Rationalisierungsprogramme zum Einsatz, etwa in Form eines Gemeinkostenmanagements (Gemeinkostenwertanalyse, Zero-Base-Budgetierung, Gemeinkosten-Systems-Engineering u.ä.). Kosten als bewerteter, sachzielbezogener Güterverzehr bieten grundsätzlich zwei Ansatzpunkte für ein Kostenniveau-Management: die Mengenkomponente und die Wertkomponente.

Die Mengenkomponente setzt unmittelbar an den Verbrauchsmengen der zum Einsatz gelangenden Güter an. Ein Instrument, das gerade im Zusammenhang mit Materialverbrauchsmengen eine breite praktische Anwendung erfährt, ist die Wertanalyse. Bei der Wertanalyse, die durch ein Denken in nutzenstiftenden Funktionen gekennzeichnet ist, handelt es sich um eine Methodik, die in systematischer Weise versucht, bei Produkten und Verfahren überflüssige Kosten zu vermeiden und dabei den Nutzen zu erhalten oder zu erhöhen. Ziel der Wertanalyse ist es somit, unter mehreren Möglichkeiten der Produkt- und Verfahrensgestaltung diejenige auszuwählen, die die niedrigsten Kosten verursacht, um so die Differenz zwischen dem Nutzen (Funktionswert oder auch ..target costs") und den dafür notwendigen Kosten zu maximieren. Eine Senkung der Kosten wird dabei über Funktions-, Produktions- und Materialkosten angestrebt. Die Wertanalyse setzt folglich primär an den Kosteneinflußgrößen Faktorqualitäten. Faktorpreise, Faktormengen und Produktionsablauf an. Weitere typische MaBnahmen eines Kostenniveau-Managements sind die Vermeidung von Doppelarbeiten und Medienbrüchen, das Ausschöpfen von Automatisierungspotentialen (besonders im indirekten Bereich) und von Spezialisierungspotentialen, die Reduzierung der Durchlaufzeiten (die häufig zwischen $80 \%$ und $90 \%$ aus Liegezeiten bestehen) und die Produktivitätssteigerung durch Gruppierung von Objekten bzw. Prozessen nach deren Ähnlichkeit (Teilefamilien- oder Losgrößenbildung). Ein wichtiges personalpolitisches Instrument zur Beeinflussung des Kostenniveaus ist das hetriebliche Vorschlagswesen. Die gezielte Generierung von kostensparenden Verbesserungsvorschlägen erfolgt dabei nicht nur durch den einzelnen Mitarbeiter, sondern wird in verstärktem Maße auch in Formen der Gruppenorganisation (Qualitätszirkel, Lernstatt, Innovationszirkel usw.) institutionalisiert. Diese Gruppenkonzepte werden mehr und mehr in das betriebliche Vorschlagswesen als "Ideenmanagement “ integriert.

Einen zweiten Ansatzpunkt für das Kostenniveau-Management bietet das Wertgerïst der Kosten. Der Beeinflussung des Kostenniveaus dienen hierbei u. a. die 
kostenorientierte Standortwahl (z. B. Verlagerung von Produktionsstätten in Niedriglohnländer), das Auffinden günstiger Bezugsquellen (z. B. „global“ sourcing und ,single" sourcing) und die Entscheidung zwischen Eigenerstellung oder Fremdbezug (make or buy) unter Berücksichtigung von Einstands- und Koordinationskosten.

\section{Kostenverlaufs-Management}

Gestaltungsobjekt dieser Maßnahmen sind die Kostenverläufe, und zwar vornehmlich das Kostenverhalten (Kostenreagibilität) in Abhängigkeit von der Beschäftigung (Outputmenge). Dabei lassen sich proportionale, progressive und degressive Kostenverläufe als Grundformen unterscheiden, die auch kombiniert auftreten können.

Eine Maßstabsfunktion für alle Bemühungen des Kostenverlaufs-Management übernimmt der proportionale bzw. lineare Kostenverlauf. Er bringt eine stetige und gleichmäßige „Normal"-Reagibilität (Reagibilitätsgrad = 1) der Kosten zum Ausdruck. Das Kostenmanagement konzentriert sich darauf, ein gleichmäßiges und nicht nur punktuell niedriges Niveau der Stückkosten herzustellen.

Bei einem progressiven Gesamtkostenverlauf nehmen die Stückkosten mit steigender Ausbringung zu (Reagibilitätsgrad $>1$ ). Verantwortlich hierfür sind beispielsweise überproportional steigende Ausschußkosten (als Folge von Ermüdungseffekten), Kosten der Terminüberschreitung (gestaffelte Konventionalstrafen) oder Koordinationskosten. Die Handhabung der Kostenprogressionen steht im Mittelpunkt des kostenorientierten Risikomanagements: Ausschlaggebend hierfür ist die durchaus plausible Befürchtung, daß eine Ausdehnung der Beschäftigung ökonomisch nicht vertretbar ist, weil die erzielbaren Nutzeneffekte durch das beschleunigte Anwachsen der Kosten überkompensiert werden.

Liegt hingegen ein degressiver Gesamtkostenverlauf vor, dann ergeben sich abnehmende Grenzkosten bei steigender Ausbringung (Reagibilitätsgrad (R): $0<R<1)$. Die Nutzung von Kostendegressionen ist Gegenstand eines Chancenmanagements, mit dessen Hilfe in erster Linie Wachstumschancen ausgeschöpft werden sollen. Derartige Kostendegressionen lassen sich zum einen durch eine Verringerung der Verbrauchsmengen, genauer eine Verringerung der Produktionskoeffizienten (Faktorverbrauchsmenge/Outputeinheit) realisieren. Technisch bedingte Gründe sind beispielsweise ein u-förmiger Verlauf der Verbrauchsfunktion, charakteristisch für den Energieverbrauch (Benzin, Strom usw.) von Aggregaten, oder die Realisation von steigenden Skalenerträgen (sog. economies of scale) durch den Einsatz von spezialisierten Fachkräften. Lernbe- 
dingte Ursachen ergeben sich aus dem Lerngesetz der (industriellen) Produktion. Danach nimmt der Zeitbedarf zur Produktion einer Einheit mit steigender (kumulierter) Ausbringungsmenge ab. Gründe hierfür sind

- beschleunigte Arbeitsabläufe, bedingt durch zunehmende Übung,

- bessere Werkzeug- und Materialhandhabung und -nutzung und

- genauere Kenntnis potentieller Störquellen (z. B. Vermeidung von Materialflußstörungen).

Auch bei der W'ertkomponente lassen sich Degressionseffekte erzielen, etwa eine durch Mengenrabatte bewirkte Senkung der Einstandspreise bei größeren Bestellmengen. Lernprozesse in allen Funktionsbereichen (Beschaffung, Produktion. Absatz usw.) bilden die Grundlage für das sog. Erfahrungskurvenkonzept, bei dem Erfahrungsvorteile in Form kombinierter Mengen- und Preiseinsparungen einen wesentlichen Einfluß auf die Wettbewerbsposition der betreffenden Unternehmung ausüben.

Fixkostenverläufe werfen das Problem der ..Null-Reagihilität " im Kostenverhalten auf: Bei gegebener Kapazität verändert sich die Kostenhöhe nicht, obwohl die Ausprägung der Kosteneinflußgröße ,Ausbringungsmenge“ variiert. Besonders wenn kapital- und damit auch fixkostenintensive Aggregate vorhanden sind, ist dafür Sorge zu tragen, da B durch hohe Outputmengen eine Fixkostendegression genutzt und damit Leerkosten vermieden werden können. Das zur Verwirklichung dieser Beschäftigungsdegression erforderliche Kapazitätsauslastungsmanagement basiert zum einen auf einer Leerzeiten minimierenden (Maschinen-)Belegungsplanung, d.h. einer Ausdehnung der effektiven Nutzungszeit von Anlagen. Hierfür eignet sich z. B. die Einführung sog. Nutzungsgradprämien. Sie belohnen die Bemühungen des Bedienungspersonals um eine Vermeidung von Stillstandszeiten, etwa durch die Durchführung kleinerer Wartungs- und Reparaturtätigkeiten. Zum anderen wird eine Ausdehnung der Betriebszeiten dieser Anlagen (z. B. durch werktägliche Schichtarbeit oder Wochenendarbeit, Pausendurchlauf bzw. .,Geisterschichten“) angestrebt.

Bei einer Fixkostenreduktion, die an der Mengenkomponente der Fixkosten ansetzt, handelt es sich entweder um eine endgültige Eliminierung Fixkosten verursachender Potentialfaktoren aus der Unternehmung (Entlassung. Verkauf, Verschrottung usw.) oder um deren Verpachtung bzw. Vermietung. Fixkostenreduktion durch Veränderung der Wertkomponente vollzieht sich über eine Senkung der Potentialfaktorpreise, etwa durch die Erschließung günstigerer Bezugsquellen.

Nicht alle Kapazitätsanpassungen schlagen sich in kontinuierlichen Kostenverläufen nieder. Diskontinuitäten in der Kostenreagibilität treten zum einen als ..Sprünge “, zum anderen als ...Knicke" in den Kostenverläufen auf. Sprung- bzw. intervallfixe Kosten werden meist dadurch verursacht, daß eine Kapazitätsan- 
passung nicht in kleinen Schritten, sondern jeweils nur in größeren Kontingenten ( $z$. B. gemessen in der vertraglich vereinbarten Arbeitszeit einer Vollzeitkraft oder der Lagerkapazität von angemieteten Lagerräumen) erfolgen kann. Diskontinuitäten in Form von „Knicken“ im Kostenverlauf (bereichsweise unterschiedlich hohe proportionale Kosten) lassen sich oft auf Unstetigkeiten in der Entwicklung des Wertgerüsts der Kosten zurückführen, beispielsweise auf eine Erhöhung der Stundenentgelte für Mehrarbeit durch die zu zahlenden Überstundenzuschlagssätze. Die Aufgabe des Kostenmanagements besteht dann in der Identifikation der jeweiligen Schwellenwerte sowie in den Bemühungen um deren Überschreitung bzw. Unterschreitung.

Spezifische Erscheinungsformen von unflexiblem Kostenverhalten ergeben sich aus der Tatsache, da $\beta$ alle Eingriffe eines Kostenmanagements, seien es nun niveau-, verlaufs- oder strukturorientierte Maßnahmen, mit einem gewissen Zeitbedarf (Erkennungszeit, Wirkungszeit usw.) verbunden sind. Durch Berücksichtigung des Zeitaspekts im Rahmen einer komparativ-statischen Kostenbetrachtung wird erkennbar, daß die Durchführung einer Kapazitätsanpassung (z. B. Einstellung eines Mitarbeiters) und die jeweils entgegengesetzte Rückführung der Kapazität zu ihrem ursprünglichen Volumen nicht zwangsläufig zu ein und derselben Kostenhöhe (bei identischem Beschäftigungsvolumen) führen. Verantwortlich für derartige Diskrepanzen ist :

- Eine Verzögerung in der Kapazitätsanpassung (infolge von Kündigungsfristen u. ä.), die etwa bewirkt, daß die Kosten zu einem bestimmten Zeitpunkt über dem erforderlichen Niveau liegen, weil es noch nicht gelungen ist, sie nach unten anzupassen. In diesem Fall ist allgemein von "Kostenremanenz" die Rede.

- Ein Vorlauf in der Kapazitätsanpassung: Hier werden Kapazitäten aufgebaut (z. B. Fachkräfte vorsorglich eingestellt), bevor sie effektiv (durch einen er-

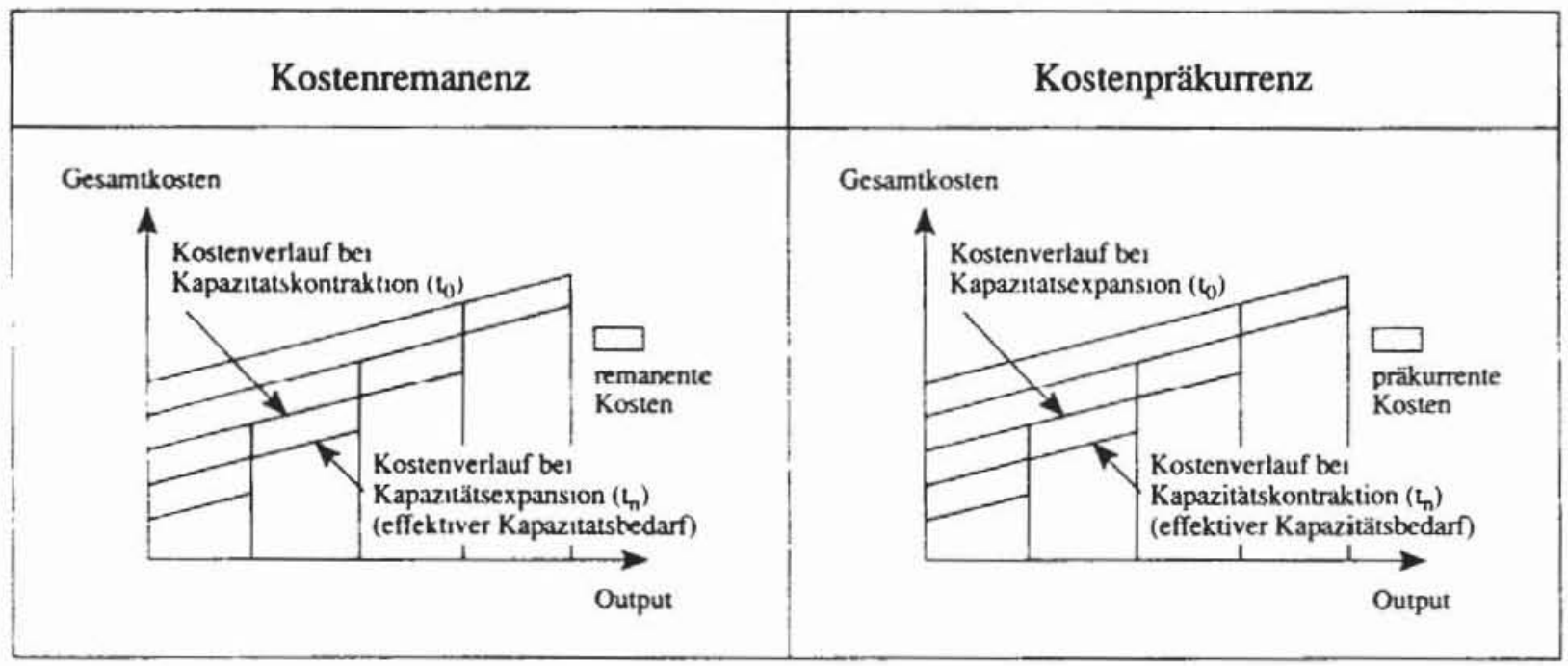

Abbildung 1: Remanenz und Präkurrenz im Kostenverlauf 
warteten, aber noch nicht erteilten Auftrag) genutzt werden können. Dieses Phänomen wird als .,Kostenpräkurrenz“" bezeichnet (vgl. Abbildung 1).

Das primäre Anliegen des Kostenmanagements besteht darin, lag-induzierte bzw. lead-induzierte Leerkosten zu vermeiden. Dies kann nur dann gelingen, wenn der jeweilige Anpassungsbedarf einerseits möglichst exakt diagnostiziert und seine Deckung damit rechtzeitig eingeleitet wird und wenn anderseits die Durchsetzung dieses Anpassungsbedarfs durch geeignete Maßnahmen der Information und Motivation abgesichert wird.

\section{Kostenstruktur-Management}

Das Management von Kostenstrukturen setzt an der Zusammensetzung der Kosten, d. h. an Mißverhältnissen bzw. Unausgewogenheiten zwischen einzelnen Kostenkategorien an. Im Zentrum der Analyse stehen dabei die beiden folgenden Strukturphänomene: Zusammensetzung der Kosten nach fixen und variablen Kosten und Zusammensetzung der Kosten nach Gemein- und Einzelkosten.

\section{Fixkosten-Strukturmanagement}

Als ungünstig werden Kostenstrukturen angesehen, die durch einen hohen Anteil an Fixkosten und einen geringen Anteil an variablen Kosten gekennzeichnet sind. Zur Handhabung fixkostenlastiger Kostenstrukturen stehen drei Vorgehensweisen zur Verfügung: die Fixkostenumwandlung. die Fixkostenumlastung sowie eine technologiebasierte Wachstumspolitik.

Bei einer Umwandlung von fixen in variable Kosten ist zu beachten, daß hierbei ceteris paribus keine Veränderung des Kostenniveaus auftritt, sondern als primäre Wirkung zunächst eine Kostenstrukturveränderung eintritt. Eine Verringerung der Kosten wird erst durch eine Beschäftigungsgradänderung bewirkt. Praktisch bedeutsame Beispiele für Fixkostenumwandlung sind: Leasing, fakultative Betriebsbereitschaft (hierbei wird ein Mietverhältnis für die Dauer des Produktionszwecks abgeschlossen, z. B. in der Bauindustrie für Baugeräte und Arbeitskräfte), Erwerb von Leistungen anderer Unternehmungen (etwa: Fremdbezug statt Eigenerstellung von Software, Zukauf von Handelsware), Leistungsentlohnung statt Zeitentlohnung. Werkverträge (Subunternehmerschaft) statt Arbeitsverträge. Diese Beispiele zeigen, da $B$ eine Fixkostenumwandlung sowohl mit Einschränkung als auch ohne Einschränkung der Produktionstiefe realisiert werden kann.

Bei der Fixkostenumlastung werden Fixkosten verursachende Produktionsfaktoren aus einem Bereich einer Unternehmung in einen anderen Bereich transfe- 
riert, der zukünftig so gut beschäftigt ist, daß in ihm eine vollständige Nutzung der betreffenden Faktorkapazitäten gewährleistet ist. Einerseits bleibt bei dieser Maßnahme das Fixkostenniveau aus der Sicht der Gesamtunternehmung konstant, anderseits wird jedoch vermieden, daß die Unternehmung eine zusätzliche Fixkostenlast auf sich nimmt, da sonst in einem Unternehmungsbereich eine Kapazitätserweiterung erfolgen müßte. Hieraus lassen sich unmittelbar die Voraussetzungen für eine Fixkostenumlastung ablesen. Neben der Flexibilität der Potentialfaktoren, und zwar sowohl in technologischer als auch in rechtlich-organisatorischer Hinsicht, z. B. Umsetzungsklauseln, müssen gleichzeitig Engpässe und Unterauslastungen in verschiedenen Unternehmungsbereichen existieren. Aus der Sichtweise des betroffenen Unternehmungsbereiches kann diese Fixkostenumlastung als Maßnahme der Fixkostenreduktion interpretiert werden.

Der technologiebasierten Wachstumspolitik liegt eine spezifische Vorstellung von technischem Fortschritt zugrunde, der sich durch Investition in ,moderne“ Technologien, d.h. in hochautomatisierte Anlagen, vollzieht (mutative Anpassung). Dieser Fortschritt geht zwar mit steigenden Fixkosten einher, die jedoch durch abnehmende variable Stückkosten überkompensiert werden. Eine Investition in Hochtechnologie erscheint allerdings nur dann ökonomisch zweckmässig, wenn im Zuge eines Wachstums die erforderliche Ausdehnung der Ausbringungsmenge sichergestellt werden kann.

\section{Gemeinkosten-Strukturmanagement}

Diese Aufgabe stellt sich für das Kostenmanagement vornehmlich im Fall einer Mehrprodukt-Produktion. Unter Einzelkosten sind dann die auf ein einzelnes Produkt zurechenbaren Kosten zu verstehen (z. B. Materialverbrauch). Demgegenüber lassen sich Gemeinkosten nur den Produktgruppen oder dem gesamten Produktionsprogramm, nicht jedoch dem einzelnen Produkt zuordnen (z. B. Grundstücke, Gebäude, Fuhrpark, Verwaltungspersonal, EDV, gemeinsame Vertriebskanäle). Ein großer Gemeinkostenblock im Vergleich zu den Einzelkosten wird dabei als ungünstig betrachtet, weil er die produktbezogene Steuerung der Unternehmungsaktivitäten beeinträchtigt. Für ein Gemeinkostenstrukturmanagement ergeben sich die folgenden Ansatzpunkte: Gemeinkostensteuerung und Gemeinkostenumwandlung.

Das Grundprinzip der Gemeinkostensteuerung ist darin zu sehen, daß die Fixkosten verursachenden Ressourcen von den einzelnen Produktbereichen als sogenannter Ressourcen-Pool genutzt werden, wobei auf der Basis geeigneter Steuerungsinstrumente dafür Sorge zu tragen ist, daß eine optimale Nutzung dieses Ressourcen-Pools erfolgt. Es geht wie im gesamtwirtschaftlichen Kontext um die optimale Allokation der Ressourcen, d. h. um die den Gesamtgewinn maxi- 
mierende Verteilung der Verfügungsrechte an knappen Ressourcen. In dieser Situation kann der Preis als flexibles Steuerungsinstrument fungieren. Das Grundprinzip einer solchen ,pretialen Lenkung“ sei an der Ressource „Finanzen“" anhand der zugrunde liegenden ,.Spielregeln“ demonstriert:

- Der zentralen Finanzabteilung kommt die Funktion eines internen Bankers zu, der das Geld den einzelnen Produktbereichen anbietet.

- Diesem Angebot an Finanzmitteln stehen die einzelnen Produktbereiche (Investment-Center) als Nachfrager gegenüber, die das Geld zur Finanzierung ihrer Investitionen benötigen.

Dieses Geld erhalten die nachfragenden Bereiche jedoch nicht kostenlos, sondern gegen Zahlung von kalkulatorischen Zinsen. Damit stellt sich die Frage, wie hoch der kalkulatorische Zinssatz. angesetzt werden sollte. Zur Beantwortung dieser Frage sind zwei Möglichkeiten gegeben. Bei der ersten Alternative verlangt die interne Finanzabteilung den Preis, den sie selbst bei Beschaffung des Geldes auf dem Kapitalmarkt dafür entrichten muB, d.h. der kalkulatorische Zinssatz richtet sich nach den effektiven Finanzierungskosten. Bei Anwendung der zweiten Alternative verlangt der interne Kapitalgeber von den nachfragenden Produktbereichen so viel, wie ihm in einer alternativen Verwendung der Finanzmittel als Zinsgewinn verlorenginge, d. h. er setzt Opportunitätskosten an. In diesem Fall ist der kalkulatorische Zinssatz so hoch wie die Rendite der besten alternativen Verwendung für dieses Geld. Damit stimmt der kalkulatorische Zinssatz mit dem internen Zinssatz der besten alternativen Verwendung überein.

Die Umwandlung von Gemeinkosten in Einzelkosten bezweckt einen prozentual möglichst geringen Gemeinkostenanteil bzw. einen möglichst hohen Einzelkostenanteil. Eine derartige Umwandlung kann dann gelingen, wenn der gemeinsam genutzte Ressourcen-Pool möglichst klein gehalten wird. Konsequenz dieser Vorgehensweise ist damit eine dezentrale Strukturierung der einzelnen Bereiche (z. B. kein zentraler, sondern ein dezentraler Vertrieb). Eine derartige Entkoppelung stellt jedoch eine teure Strategie dar: Sie läuft auf eine Parallelausstattung der einzelnen Sparten mit gleichartiger Personal-, EDV-, Vertriebskapazität usw. hinaus, da nur so die erforderliche Autarkie (Verfügung über eigene Ressourcen) der betroffenen Einheiten hergestellt werden kann.

\section{Optimierung von Kostenstrukturen}

Grundgedanke dieser Strategie ist es, da $B$ weder eine radikale Fixkosten- noch eine Gemeinkostenumwandlung mit dem Ziel der Fixkosten- bzw. Gemeinkostenminimierung empfehlenswert ist, da mit dem Abbau eines Fix- bzw. Gemeinkostenblocks auch gewichtige Vorteile verlorengehen können (vgl. Abbildung 2): 
- Die Fixkosten verursachenden Potentialfaktoren bieten den Vorteil von Sicherheitsreserven, sofern sie nicht vollständig ausgelastet sind. Derartige Slack-Ressourcen sind ein wertvolles Instrument des Risikomanagements, wenn es gilt, eine unerwartete Spitze in der Beschäftigung (z. B. einen Zusatzauftrag) zu bewältigen oder den plötzlichen Ausfall von Aggregaten, Lieferungen oder Mitarbeitern (Fehlzeiten) zu kompensieren.

- Gemeinkosten verursachende gepoolte Ressourcen bieten Größenvorteile. Sie entstehen dadurch, da $B$ etwa auf dem Beschaffungsmarkt durch gemeinsamen Einkauf aller Produktbereiche Nachfragemacht ausgeübt werden kann. Ressourcenpools entfalten Synergieeffekte bei der Produktion unterschiedlicher Güter, sofern diese eine produktionstechnische Verwandtschaft aufweisen. So können die Gesamtkosten der Produktion zweier unterschiedlicher Produkte ( z. B. Lastkraftwagen und Omnibusse) niedriger sein als die Summe der Produktionskosten für die beiden Güter $\left(\mathrm{K}\left(\mathrm{x}_{1}, \mathrm{x}_{2}\right)<\mathrm{K}\left(\mathrm{x}_{1}\right)+\mathrm{K}\left(\mathrm{x}_{2}\right)\right)$. Dieses Phänomen, das auf einer gemeinsamen, dabei aber nicht rivalisierenden Nutzung von Potentialen beruht (z. B. ein F\&E-Mitarbeiter bringt sein Know how in beide Produktionsprozesse ein), wird als ,economies of scope“ bezeichnet.

Die Bemühungen um eine simultane Optimierung von Fixkosten- und Gemeinkostenstruktur erlauben es zudem, eine Brücke zwischen dem Kostenmanagement und einer kostenorientierten Wachstumspolitik zu schlagen. Wachstum nur mit Blick auf die Fixkostenstruktur ist gewissermaßen auf Degressionseffekte (Skaleneffekte mit oder ohne technischen Fortschritt) „fixiert". Eine Relativierung erfährt diese Wachstumsphilosophie des ,,big is beautiful“" nicht zuletzt durch die Progressionseffekte der Koordinationskosten: Positive und negative Skaleneffekte (.,economies“ und ,diseconomies of scale“) zusammen münden in die Vorstellung von einer optimalen Betriebsgröße.

Für eine Horizonterweiterung sorgt nun die Einbeziehung der Gemeinkostenstruktur in die Wachstumspolitik. Durch eine konsequente Dezentralisation lassen sich, wie erläutert, die Gemeinkosten und die Koordinationskosten zwischen den dezentral agierenden Organisationseinheiten verringern. Voraussetzung

\begin{tabular}{|l|l|l|}
\hline $\begin{array}{r}\text { Bewertungs- } \\
\text { aspekt }\end{array}$ & Kostenaspekt & Nutzenaspekt \\
\hline $\begin{array}{l}\text { Ressourcenpotentiale } \\
\text { (eingeschränkte Teilbarkeit) }\end{array}$ & Fixkosten & Slack \\
\hline $\begin{array}{l}\text { Ressourcenpoolung } \\
\text { (eingeschränkte Zurechenbarkeit) }\end{array}$ & Gemeinkosten & Synergie \\
\hline
\end{tabular}

Abbildung 2: Bewertung von Kostenstrukturen 
hierfür ist allerdings ein gewisses $\mathrm{Maß}$ an Autarkie der betreffenden Landesgesellschaften, Sparten, Werke. Fertigungssegmente, Abteilungen usw. Das Wachstum vollzieht sich dann in dem Sinne "modular", daß die Gesamtbetriebsgröße zunimmt, ohne daß die Größe der einzelnen organisatorischen Teilbereiche (Module) awangsläufig ebenfalls zunehmen müßte. Da aber im Zuge der Entkopplung (Modularisierung) in den Organisationseinheiten zusätzliche Kapazitäten aufgebaut werden, geht die Verringerung der Gemeinkosten in aller Regel Hand in Hand mit einer Erhöhung der Unternehmungs-Fixkosten (Leerkosten): Modulare Unternehmungsstrukturen beschneiden u. a. das Spektrum der Umlastungsalternativen.

\section{Fokussiertes Kostenmanagement}

Niveau-, Verlaufs- und Strukturmanagement bilden die Säulen eines globalen Kostenmanagements. Um nun zu verhindern, daß die .,Kosten des Kostenmanagements" außer Kontrolle geraten, erscheint eine bewußte Konzentration auf ausgewählte Kostenkategorien unausweichlich: Anstatt sich flächendeckend um alle Kostenkategorien mit gleicher Aufmerksamkeit zu kümmern, setzt das fokussierte Kostenmanagement nur an den Hauptverursachern und dabei jeweils an den Wurzeln der Kostenverursachung an. Die Frage, ob nun die Transportkosten oder eher die hohen Lagerbestände, ob die Forschungs- und Entwicklungsaktivitäten, aufgetretene Qualitätsmängel (Rückrufaktionen usw.). die EDV-Kosten oder die Personalkosten, getrennt nach Direktentgelten und Sozialleistungen, die hauptsächlichen und eigentlichen Kostentreiber darstellen. läßt sich nur beantworten auf der Basis einer aussagefähigen Kostenklassifikation. Die geläufigen Kostenaufgliederungen aus der Kostenarten-. Kostenstellen- und Kostenträgerrechnung bieten hierfür eine geeignete Ausgangsbasis.

Einen weiteren Ansatzpunkt für ein fokussiertes Kostenmanagement bietet die aus der Aktivitätskostenrechnung bekannte Unterteilung in value und nonvalue activities. Bei letzteren handelt es sich um Aktivitäten, die mit keinem unmittelbaren betrieblichen Wertzuwachs einhergehen. Lassen sich derartige Aktivitäten identifizieren, dann sind diese einzuschränken oder in vollem Umfang abzubauen. Zu Fokussierungszwecken ist diese qualitative Klassifikation durch eine quantifizierte Kategorisierung zu ergänzen, die Auskunft über die quantitativen Anteile der einzelnen Kostenkategorien am Gesamtkostenvolumen gibt. Hierzu eignet sich eine ABC-Analyse der Kostenverursacher. Sie ermöglicht als Screening-Verfahren eine erste Identifikation der Kostenkategorien bzw. -determinanten (A-Kosten), die für einen hohen Kostenanteil verantwortlich sind und auf die die kostenpolitischen Interventionen zunächst auszurichten sind. Dabei sei betont, da B die Einteilung in A-, B- und C-Klassen eher willkürlich ist und eine entscheidungstheoretisch fundierte Abgrenzung dieser Klassen mit erheblichen Schwierigkeiten einhergeht. 
Klassifikation allein bildet allerdings keine ausreichende Basis für ein fokussiertes Kostenmanagement: Die Identifikation einzelner Kostentreiber verleitet leicht zu punktuellen Kosteninterventionsprogrammen. Derartige klein-formatierte Eingriffe werden aber den vielfältigen, zwischen den verschiedenen Kostenkategorien bestehenden Verbundbeziehungen nicht gerecht: So macht es wenig Sinn, die Kosten eines Produkts schwerpunktmäßig im Fertigungsbereich anzugehen, wenn bereits $70 \%$ der Kosten durch Entscheidungen in der Konstruktionsphase determiniert wurden. Ähnlich irreführend wirkt die Diagnose hoher Montagekosten anhand einer Kostenzuwachskurve über die Stufen der Leistungserstellung, wenn lange Montagezeiten nichts anderes darstellen als die Fortpflanzung eines Fehlers in der vorgelagerten Teilefertigung. Parallel zur Kostenklassifikation muß also eine Analyse der Verbundbeziehungen zwischen den einzelnen Kostenkategorien vorgenommen werden. Das jeweils der Planung zugrundeliegende Sukzessionsprinzip dient als Wegweiser bei der Suche nach geeigneten Ansatzpunkten für ein Kostenmanagement. Das gilt sicherlich für den Verbund im Rahmen einer mehrstufigen Leistungserstellung. Es gilt gleichermaßen für andere Induktionsvorgänge, beispielsweise bei der Faktorkombination (Kostenartenverbund, z. B. höhere Qualifikationsanforderungen im Gefolge von hochautomatisierten Anlagen).

\section{Gestaltungsfunktionen: Diagnose, Planung und Implementierung}

Das Kernstück des Kostenmanagements bildet die Kostenplanung. Sie legt zum einen als Zielplanung eine punktuell fixierte Kostenhöhe oder eine Obergrenze für die Kosten fest. Derartige Soll-, Plan- bzw. Budgetgrößen werden als Kostenvorgaben bzw. Kostenvereinbarungen formuliert. Zum anderen bestimmt sie als Maßnahmenplanung die jeweiligen Eingriffe auf der Ebene der Kosteneinflußgrößen. Hierzu zählen beispielsweise kostenreduzierende Maßnahmen der Personalumsetzung, des Personalabbaus, der Einschränkung des Variantenspektrums, des Verfahrenswechsels bzw. der Verringerung der Fertigungstiefe.

Allerdings wäre es falsch, das Kostenmanagement auf die Kostenplanung zu reduzieren. Die Planung der Kosten ist vielmehr eingebettet in vor- und nachgelagerte Managementaktivitäten, die einen ebenso wichtigen Einfluß auf den Erfolg des Kostenmanagements besitzen. In diesem Zusammenhang ist zunächst die traditionelle Kostenkontrolle zu nennen. Sie informiert über SollIst-Abweichungen und führt diese Abweichungen im Rahmen einer Abweichungsanalyse anteilig auf bestimmte Verursachungsfaktoren (Preise, Verbrauchsmengen. Beschäftigungsgrade usw.) zurück. Auf dem Wege einer 
Rückmeldung der Abweichungen signalisiert sie Notwendigkeiten für eine Änderung oder Anpassung. sprich für Handlungsbedarfe in der nachfolgenden Planungsperiode. Solche Änderungs- bzw. Eingriffsbedarfe lassen sich jedoch nicht nur nachträglich konstatieren. Sie lassen sich auch antizipieren, bevor sie tatsächlich eingetreten sind. Dazu bedarf es einer auf Prognosen basierenden Ermittlung von Soll-Wird-Abweichungen. Im allgemeinen ist hier von der Problemerkennung, d.h. der Erkennung von Kostenproblemen, die Rede. Im Rahmen eines Managements der Qualitätskosten wird in der Praxis beispielsweise davon ausgegangen. daß die Kosten jeweils um das Zehnfache steigen, wenn ein Qualitätsmangel nicht bereits in der Konstruktion, in der Fertigung, in der Ausgangskontrolle usw., sondern erst beim Kunden erkannt wird. Soll-IstVergleiche (Kostenkontrolle) und Soll-Wird-Vergleiche (Problemerkennung) dienen beide der Erkennung von Handlungsbedarfen. Für die sich mit Handlungsbedarfen befassende Managementfunktion hat sich die Bezeichnung „Diagnose" eingebürgert. Kostenmanagement sollte neben der Kostenplanung auch ein funktionstüchtiges Diagnose-Modul umfassen.

Kostenpolitische Eingriffe müssen nicht nur geplant, sie müssen auch implementiert werden. Es reicht nicht aus, den kostenminimalen Personalbestand oder die optimale Fertigungstiefe zu planen. Derartigen Planungsbemühungen ist nur dann Erfolg beschieden. wenn sie von den Betroffenen (Kostenstellenleiter. Betriebsrat, Mitarbeiter, Lieferanten usw.) akzeptiert werden. Ansonsten muß mit allen Spielarten von Opposition gerechnet werden, von der passiven Leistungszurückhaltung bis zu manifesten Widerständen. Implementierung von kostenreduzierenden Maßnahmen ist dann erfolgreich, wenn die Betroffenen die notwendigen Fähigkeiten sowie die Bereitschaft zur Umsetzung dieser Maßnahmen besitzen. Implementierungsaktivitäten in Form von Informationsaktionen, Qualifikationsprogrammen, Motivierungs- und Partizipationsmaßnahmen verursachen wiederum Kosten. Diese Kosten eines konsequent betriebenen Kostenmanagements müssen den Opportunitätskosten einer unterlassenen Implementierung gegenübergestellt werden.

Aus diesen Überlegungen wird ersichtlich, da $B$ das Kostenmanagement insgesamt als Gestaltungsobjekt eines Meta-Kostenmanagements anzusehen ist. Neben den bereits genannten Kosten des Kostenmanagements geht in die Kostenbetrachtung ein gewaltiger Kostenblock ein: die Kosten der Kostenrechnungssysteme. Diese Kostenrechnungssysteme bilden ja das unverzichtbare informatorische Fundament für alle Domänen des Kostenmanagements. 


\section{Literaturhinweise}

ADAM, Dietrich: Kostendegressionen und -progressionen, in: Handwörterbuch der Produktionswirtschaft, hrsg. von Kern, Werner, Stuttgart 1979, Sp. 939-955; BAUER, HaNS: Das Erfahrungskurvenkonzept, Möglichkeiten und Problematik der Ableitung strategischer Handlungsalternativen, in: Wirtschaftswissenschaftliches Studium, 15. Jg. (1986), S. 1-10; BAUR, WALTER: Lerngesetz der industriellen Produktion, in: Handwörterbuch der Produktionswirtschaft, hrsg. von Kern, Werner, Stuttgart 1979, Sp. 1115-1125; Berginer, Heinz: Der Ersatz fixer Kosten durch variable Kosten, in: Zeitschrift für betriebswirtschaftliche Forschung, 19. Jg. (1967), S. 141 -162; CoRsten, Hans: Fixkostenabbau bei schrumpfenden Unternehmungen, in: Das Wirtschaftsstudium, 14. Jg. (1985), S. 531-536; GäLwEILER, AlOYS: Steuerung der Kostenhöhe und der Kostenstruktur durch strategische Planung, in: Die Betriebswirtschaft, 37. Jg. (1977), S. 67-75; Gans, Christian - Michael Reiss: Diagnostisches Rechnungswesen: Die Nutzung der Kostenrechnung zur Erkennung betrieblicher Handlungsnotwendigkeiten, in: Kostenrechnungspraxis, (1986), S. 95-100; KERN, WERNER: Industrielle Produktionswirtschaft, 4. Aufl., Stuttgart 1990; KunZ, Beat Rudolf: Die Remanenz der Kosten eines Betriebes, in: Wirtschaftswissenschaftliches Studium, 11. Jg. (1982), S. 20-24; KüPPER, HANS-ULRICH: Kosten, fixe und variable, in: Handwörterbuch des Rechnungswesens, hrsg. von Chmielewicz, Klaus u. a., 2. Aufl., Stuttgart 1981, Sp. 950-962; Reiss, Michael: Der Zeitbezug des betrieblichen Rechnungswesens, in: Das Wirtschaftsstudium, 16. Jg. (1987), S. 429-435; SüvERKRÜP, FRITZ: Die Abbaufähigkeit fixer Kosten. Unternehmenspolitische Möglichkeiten ihrer Beherrschung, Berlin 1968; WEBER, JÜRGEN: Variable und fixe Kosten. Eine überflüssige Unterteilung der Kosten?, in: Wirtschaftswissenschaftliches Studium, 16. Jg. (1987), S. 393-398. 